\title{
Lipschitz symmetric functions on Banach spaces with symmetric bases
}

\author{
Martsinkiv M.V., Vasylyshyn S.I., Vasylyshyn T.V. ${ }^{\bowtie}$, Zagorodnyuk A.V.
}

We investigate Lipschitz symmetric functions on a Banach space $X$ with a symmetric basis. We consider power symmetric polynomials on $\ell_{1}$ and show that they are Lipschitz on the unbounded subset consisting of vectors $x \in \ell_{1}$ such that $\left|x_{n}\right| \leq 1$. Using functions max and min and tropical polynomials of several variables, we constructed a large family of Lipschitz symmetric functions on the Banach space $c_{0}$ which can be described as a semiring of compositions of tropical polynomials over $c_{0}$.

Key words and phrases: Lipschitz symmetric function on Banach space, symmetric basis, tropical polynomial.

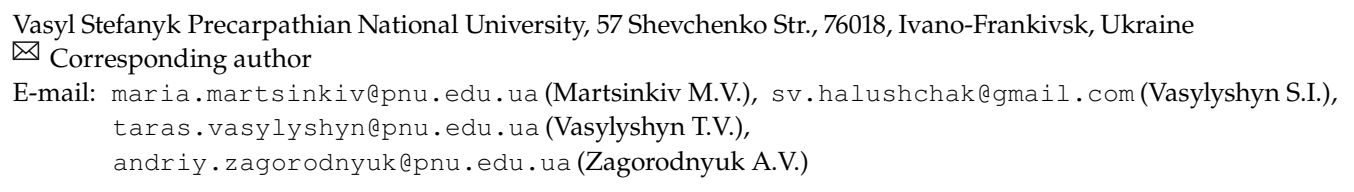

\section{Introduction}

Symmetric functions of infinitely many variables play an important role in the nonlinear functional analysis and its applications [12]. Let $X$ be a real Banach space. We recall that a Schauder basis $\left(e_{n}\right)$ in $X$ is symmetric if it is equivalent to the basis $\left(e_{\sigma(n)}\right)$ for every permutation $\sigma$ of the set of positive integers $\mathbb{N}$. Let us denote by $\mathcal{S}^{\infty}$ the group of all permutations (bijections) on the set of natural numbers $\mathbb{N}$. Any $\sigma \in \mathcal{S}^{\infty}$ acts on $X$ by

$$
\sigma(x)=\left(x_{\sigma(1)}, \ldots, x_{\sigma(n)}, \ldots\right), \quad x=\sum_{n=1}^{\infty} x_{n} e_{n}=\left(x_{1}, \ldots, x_{n}, \ldots\right) \in X .
$$

A function $f: X \rightarrow \mathbb{R}$ is called symmetric if $f(\sigma(x))=f(x)$ for all $x \in X$ and $\sigma \in \mathcal{S}^{\infty}$. It is naturally to study symmetric polynomials and analytic functions on $X$ as "simple" nonlinear symmetric functions. Algebras of symmetric analytic functions and their generalizations on real and complex Banach spaces were investigated by many authors (see [1-9, 14, 16-18] and references therein). However, some Banach spaces like $c_{0}$ do not support symmetric polynomials while support a lot of symmetric Lipschitz functions. In [12] it was proposed symmetric slice polynomials for approximation of uniformly continuous symmetric functions on $c_{0}$. But the slice polynomials are not Lipschitz in the general case. In this paper we consider some classes of Lipschitz symmetric functions on a real Banach space $X$ with a symmetric basis.

$\mathrm{y} \Delta \mathrm{K} 517.98$

2020 Mathematics Subject Classification:41A65, 46E15, 46G25, $05 \mathrm{E} 05$.

This research was funded by the National Research Foundation of Ukraine, 2020.02/0025, 0121U111037 
A mapping $P$ from a Banach space $X$ to a Banach space $Y$ is an $n$-homogeneous polynomial if there is an $n$-linear map $B$ on the $n$th Cartesian power $X^{n}$ to $Y$ such that $P(x)=B(x, \ldots, x)$. A finite sum of homogeneous polynomials $P=P_{0}+P_{1}+\cdots+P_{m}$ is a polynomial of degree $m$ if each $P_{n}$ is an $n$-homogeneous polynomial, $0 \leq n \leq m$, and $P_{m} \not \equiv 0$.

Let us recall that a function $f$ from a metric space $\left(M_{1}, \rho_{1}\right)$ to a metric space $\left(M_{2}, \rho_{2}\right)$ is Lipschit $z$ if there is a constant $L$ such that $\rho_{2}(f(x), f(y)) \leq L \rho_{1}(x, y), x, y \in M_{1}$. The infimum over all constants $L$ satisfying the inequality above is called the Lipschitz constant of $f$ and denoted by $L(f)$. We refer the reader to the N. Weaver book [19] for details about Lipschitz mappings and to J. Mujica book [13] for details on polynomials and analytic functions on Banach spaces.

In Section 1, we discuss the question about existence of Lipschitz symmetric polynomials on an unbounded domain in $\ell_{1}$. In Section 2 , we consider Lipschitz functions on $X$, constructed using operations $\max$, min and their linear combinations. It leads us to so-called Tropical Mathematics [15] which proceeds with semirings involving such operations. Some connections between Lipschitz symmetric functions on $X$ and tropical polynomials of infinitely many variables are found.

\section{Lipschitz symmetric polynomials}

It is clear that any polynomial of degree greater than 1 is not Lipschitz on $X$, even if $X$ is finite dimensional. However polynomials are locally Lipschitz. Among of symmetric polynomials we can find Lipschitz ones on some unbounded sets.

Let $c_{00}$ be the space of all finite sequences, that is, if $x=\left(x_{1}, \ldots, x_{n}, \ldots\right) \in c_{00}$, then only a finite number of coordinates $x_{n}$ is not equal to zero. We consider power symmetric polynomials

and elementary symmetric polynomials

$$
F_{m}(x)=\sum_{i=1}^{\infty} x_{i}^{m}
$$

$$
G_{m}(x)=\sum_{i_{1}<\cdots<i_{m}} x_{i_{1}} \cdots x_{i_{m}}, \quad m \in \mathbb{N}, \quad x \in c_{00} .
$$

It is well known that polynomials $\left(F_{m}\right)$ and $\left(G_{m}\right)$, form algebraic bases in the algebra of all symmetric polynomials on $c_{00}$ (see e.g. [11]). Both $\left(F_{m}\right)$ and $\left(G_{m}\right)$ can be extended to $\ell_{1}$ for every $m \in \mathbb{N}$. We will use the same symbols for the extensions.

Lemma 1. Symmetric polynomials $F_{m}^{k}(x)=\left(\sum_{i=1}^{n} x_{i}^{m}\right)^{k}$ on $\mathbb{R}^{n}$ with the $\ell_{1}$-norm are Lipschitz with constants $n^{k-1} \leq L\left(F_{m}^{k}\right) \leq m k n^{k-1}$ on the domain $D_{n}=\left\{x \in \mathbb{R}^{n}:\left|x_{i}\right| \leq 1, i=1, \ldots, n\right\}$.

Proof. We have

$$
\begin{aligned}
\left|F_{m}^{k}(x)-F_{m}^{k}(y)\right|= & \left|F_{m}(x)-F_{m}(y)\right| \mid F_{m}^{k-1}(x)+F_{m}^{k-2}(x) F_{m}(y) \\
& +\ldots+F_{m}(x) F_{m}^{k-2}(y)+F_{m}^{k-1}(y) \mid \\
\leq & \sum_{i=1}^{n}\left|x_{i}^{m}-y_{i}^{m}\right| \mid\left(\sum_{i=1}^{n} x_{i}^{m}\right)^{k-1}+\left(\sum_{i=1}^{n} x_{i}^{m}\right)^{k-2} \sum_{i=1}^{n} y_{i}^{m} \\
& +\ldots+\sum_{i=1}^{n} x_{i}^{m}\left(\sum_{i=1}^{n} y_{i}^{m}\right)^{k-2}+\left(\sum_{i=1}^{n} y_{i}^{m}\right)^{k-1} \mid .
\end{aligned}
$$


Since $\left|x_{i}\right| \leq 1$ and $\left|y_{i}\right| \leq 1$, it follows that

$$
\begin{aligned}
\left|F_{m}^{k}(x)-F_{m}^{k}(y)\right| & \leq k n^{k-1} \sum_{i=1}^{n}\left|x_{i}^{m}-y_{i}^{m}\right| \\
& =k n^{k-1} \sum_{i=1}^{n}\left|\left(x_{i}-y_{i}\right)\left(x_{i}^{m-1}+x_{i}^{m-2} y_{i}+\cdots+x_{i} y_{i}^{m-2}+y_{i}^{m-1}\right)\right| \\
& \leq m k n^{k-1} \sum_{i=1}^{n}\left|x_{i}-y_{i}\right|=m k n^{k-1}\|x-y\|_{\ell_{1}} .
\end{aligned}
$$

To get a lower estimation, we set $x_{0}=(\underbrace{1, \ldots, 1}_{n})$ and $y_{0}=0$. Then

$$
\left|F_{m}^{k}(x)-F_{m}^{k}(y)\right| \geq\left|F_{m}^{k}\left(x_{0}\right)-F_{m}^{k}\left(y_{0}\right)\right|=n^{k}=n^{k-1}\left\|x_{0}-y_{0}\right\|_{\ell_{1}} .
$$

Since all norms on $\mathbb{R}^{n}$ are equivalent, we have that $F_{m}^{k}$ are Lipschitz functions for any norm on $\mathbb{R}^{n}$. But for the case of $\ell_{1}$-norm we have estimations for the Lipschitz constant which do not depend on $n$ if $k=1$. Thus we can prove the following theorem.

Theorem 1. Polynomials $F_{m}, m \in \mathbb{N}$ are Lipschitz functions on $D_{\infty}=\left\{x \in \ell_{1}:\left|x_{i}\right| \leq 1, i \in \mathbb{N}\right\}$ with $1 \leq L\left(F_{m}\right) \leq m$ and $F_{m}^{k}$ are not Lipschitz on $D_{\infty}$ for every $k>1$.

Proof. Since the estimation $1 \leq L\left(F_{m}\right) \leq m$ holds for every $\left(\mathbb{R}^{n},\|\cdot\|_{\ell_{1}}\right)$, it is still correct if $n \rightarrow \infty$. For $k>1$ we have that $n^{k-1} \leq L\left(F_{m}^{k}\right)$ and so $L\left(F_{m}^{k}\right) \rightarrow \infty$ as $n \rightarrow \infty$.

Note that polynomials $G_{m}$ are not Lipschitz on $D_{\infty}$. For example, routine calculations show that for the Lipschitz constant of the restriction of $G_{2}$ to $D_{2}$ we have $(n-1) / 2 \leq L\left(G_{2}\right) \leq n-1$.

\section{Banach spaces and tropical semirings of Lipschitz functions}

It is well-known (see, e.g., [10, p. 114]) that, on every Banach space with a symmetric basis, there is an equivalent symmetric norm $\|\cdot\|$ and

$$
\|x\|=\left\|\sum_{n=1}^{\infty} x_{n} e_{n}\right\|=\left\|\sum_{n=1}^{\infty}\left|x_{n}\right| e_{n}\right\|, \quad x \in X .
$$

Throughout this section we suppose that the real Banach space $X$ has a symmetric basis $\left(e_{\sigma(n)}\right), n \in \mathbb{N}$, is endowed with a symmetric norm $\|\cdot\|=\|\cdot\|_{X}$, and the $c_{0}$-norm is continuous on $X$, that is there is a constant $C>0$, such that $\|x\|_{c_{0}}=\sup _{n}\left|x_{n}\right| \leq C\|x\|_{X}$ for every $x=$ $\left(x_{1}, \ldots, x_{n}, \ldots\right) \in X$. Spaces $c_{0}$ and $\ell_{p}$, for $1 \leq p<\infty$ are typical examples of such spaces. Let $\mathbb{Z}_{0}=\mathbb{Z} \backslash\{0\}$. We will use notation $X\left(\mathbb{Z}_{0}\right)$ for the "two-side $X$ ", that is $X \oplus X$ indexed by negative and positive integers.

Let $x=\left(x_{1}, x_{2}, \ldots, x_{n}, \ldots\right) \in X$. We denote by $\operatorname{supp}^{+}(x):=\left\{k \in \mathbb{N}: x_{k}>0\right\}$ and by $\operatorname{supp}^{-}(x):=\left\{k \in \mathbb{N}: x_{k}<0\right\}$. Clearly, $\operatorname{supp}(x)=\operatorname{supp}^{+}(x) \cup \operatorname{supp}^{-}(x)$. For every vector $x \in X$ we assign a vector $\widehat{x}=\left(\ldots, 0, \widehat{x}_{-j}, \ldots, \widehat{x}_{-1}, \widehat{x}_{1}, \ldots, \widehat{x}_{m}, 0, \ldots\right)$ in the space $X\left(\mathbb{Z}_{0}\right)$, ordered by the following way: $m=\left|\operatorname{supp}^{+}(x)\right|, j=\left|\operatorname{supp}^{-}(x)\right|$ ( $m$ and/or $j$ may be equal to infinity) and $\widehat{x}_{-j} \leq \cdots \leq \widehat{x}_{-1}$, and $\widehat{x}_{1} \geq \cdots \geq \widehat{x}_{m}$.

We denote by $\mathcal{M}_{X} \subset X\left(\mathbb{Z}_{0}\right)$ the set $\{\widehat{x}: x \in X\}$. The set $\mathcal{M}_{X}$ can be considered as the quotient of $X$ with respect to the following equivalence: $x \sim y$ if and only if $\widehat{x}=\widehat{y}$. We suppose that $\mathcal{M}_{X}$ is endowed with the quotient topology, that is, the strongest topology such that the quotient map $x \mapsto \widehat{x}$ is continuous. 
Proposition 1. Let $Y$ be a topological space and $f: \mathcal{M}_{X} \rightarrow Y$ be a continuous map. Then $\check{f}(x):=f(\widehat{x})$ is a symmetric and continuous map on $X$.

Proof. The continuity of $\check{f}$ follows from the fact that the quotient map is open. Also, by the definition of $f, f(x)=f(y)$ if $x \sim y$.

For a given $\widehat{x}=\left(\ldots, 0, \widehat{x}_{-j}, \ldots, \widehat{x}_{m}, 0, \ldots\right)$ and every $n \in \mathbb{Z}_{0}$ we define

$$
g_{n}(x)= \begin{cases}\widehat{x}_{n}, & \text { if }-j \leq n \leq m \\ 0, & \text { otherwise }\end{cases}
$$

Theorem 2. Functions $g_{n}$ are Lipschitz symmetric on $X$ with $1 \leq L\left(g_{n}\right) \leq C$, where $C>0$ is such that $\|u\|_{c_{0}} \leq C\|u\|_{X}, u \in X$. If $\widehat{x}=\left(\ldots, 0, \widehat{x}_{-j}, \ldots, \widehat{x}_{m}, 0, \ldots\right)$, then

$$
g_{n}(x)=\max _{i_{1}<\cdots<i_{n}}\left(\min \left(\widehat{x}_{i_{1}}, \ldots, \widehat{x}_{i_{n}}\right)\right) \text { for } n>0,
$$

and

$$
g_{n}(x)=\min _{i_{1}<\cdots<i_{n}}\left(\max \left(\widehat{x}_{-i_{1}}, \ldots, \widehat{x}_{-i_{n}}\right)\right) \quad \text { for } n<0 .
$$

Proof. The symmetry of $g_{n}$ follows from Proposition 1. Equation (1) is correct because if $0<$ $n \leq m$, then $\max _{i_{1}<\cdots<i_{n}}\left(\min \left(\widehat{x}_{i_{1}}, \ldots, \widehat{x}_{i_{n}}\right)\right)$ will be attended at the tuple $\left(\widehat{x}_{1}, \widehat{x}_{2}, \ldots, \widehat{x}_{n}\right)$ and will be equal to $\widehat{x}_{n}$. If $n>m$, then each tuple $\left(\widehat{x}_{1}, \widehat{x}_{2}, \ldots, \widehat{x}_{n}\right)$ will contain 0 and so $g_{n}(x)=0$. Formula (2) can be proved in a similar way.

Let us show that $g_{n}$ is a Lipschitz function for $n>0$. Let $x, y \in X$ and $k, i \in \mathbb{N}$ are such that $x_{k}=\widehat{x}_{n}$ and $y_{i}=\widehat{y}_{n}$. Without loss of the generality, we can assume that $x_{k} \geq y_{i}$. Consider the case $n=1$. Since $g_{1}(y)=y_{i}$, it follows that $y_{i} \geq y_{s}$ for every $s \in \mathbb{N}$. In particular, $y_{i} \geq y_{k}$. Thus, we have $x_{k} \geq y_{i} \geq y_{k} \geq 0$. Consequently,

$$
\left|g_{1}(x)-g_{1}(y)\right|=\left|x_{k}-y_{i}\right| \leq\left|x_{k}-y_{k}\right| \leq\|x-y\|_{c_{0}} \leq C\|x-y\|_{X} .
$$

Consider the case $n>1$. Since $g_{n}(x)=x_{k}$, it follows that there exists the set of indexes $\left\{s_{1}, \ldots, s_{n}\right\}$ such that $x_{s_{1}} \geq x_{k}, \ldots, x_{s_{n}} \geq x_{k}$. Since $g_{n}(y)=y_{i}$, it follows that there exists not more than $n-1$ indexes $m \in \mathbb{N}$ such that $y_{m}>y_{i}$. Therefore, taking into account that $\left|\left\{s_{1}, \ldots, s_{n}\right\}\right|=n>n-1$, there exists $j \in\{1, \ldots, n\}$ such that $y_{s_{j}} \leq y_{i}$. Thus, we have $x_{s_{j}} \geq x_{k} \geq y_{i} \geq y_{s_{j}} \geq 0$. Consequently,

$$
\left|g_{n}(x)-g_{n}(y)\right|=\left|x_{k}-y_{i}\right| \leq\left|x_{s_{j}}-y_{s_{j}}\right| \leq\|x-y\|_{c_{0}} \leq C\|x-y\|_{X}
$$

In the case $n<0$ the proof is analogical.

Note that the functions $g_{n}$ are nonlinear and $g_{1}(x)=\sup _{k} x_{k}$. It can be checked that we have the following formula for representation of any element in $\mathcal{M}_{X}$.

Proposition 2. Every $\widehat{x} \in \mathcal{M}_{X} \subset X\left(\mathbb{Z}_{0}\right)$ can be represented by

$$
\widehat{x}=\sum_{n \in \mathbb{Z}_{0}} g_{n}(x) e_{n} .
$$

Theorem 2 and Proposition 2 imply the following corollary.

Corollary 1. The mapping $\iota: c_{0} \ni x \mapsto \widehat{x} \in \mathcal{M}_{c_{0}}$ is 1-Lipschitz. 
Proof. By Proposition 2,

$$
\|\widehat{x}-\widehat{y}\|=\left\|\sum_{n \in \mathbb{Z}_{0}}\left(g_{n}(x)-g_{n}(y)\right) e_{n}\right\|_{c_{0}}=\sup _{n \in \mathbb{Z}_{0}}\left|g_{n}(x)-g_{n}(y)\right| .
$$

By Theorem 2, $L\left(g_{n}\right)=1$ for the case $X=c_{0}$. Therefore $\left|g_{n}(x)-g_{n}(y)\right| \leq\|x-y\|_{c_{0}}$. Consequently, $\|\widehat{x}-\widehat{y}\| \leq\|x-y\|_{c_{0}}$.

Let $\varphi \in c_{0}\left(\mathbb{Z}_{0}\right)^{*}$ be a linear continuous functional on $c_{0}\left(\mathbb{Z}_{0}\right)$. Then $\varphi$ is completely defined by the sequence of its values on the basis vectors, $\left(c_{n}\right)=\left(\varphi\left(e_{n}\right)\right) \in \ell_{1}\left(\mathbb{Z}_{0}\right)$. In this sense, we will say that $c_{0}\left(\mathbb{Z}_{0}\right)^{*}$ coincides with $\ell_{1}\left(\mathbb{Z}_{0}\right)$.

Corollary 2. For every $\varphi=\left(c_{n}\right) \in \ell_{1}\left(\mathbb{Z}_{0}\right)$ the function

$$
g_{\varphi}(x):=\sum_{n \in \mathbb{Z}_{0}} c_{n} g_{n}(x)
$$

is a Lipschitz symmetric function and $L\left(g_{\varphi}\right) \leq L(\varphi)$.

Proof. According to Proposition 2, we have that $g_{\varphi}(x)=\varphi(\widehat{x})$ and so it is well-defined. From Corollary 1 it follows that $g_{\varphi}=\varphi \circ \iota$ is a composition of two Lipschitz mappings and so it is Lipschitz with $L\left(g_{\varphi}\right) \leq L(\iota) L(\varphi)=L(\varphi)[19$, p. 4].

Let us estimate the norm of $g_{\varphi}$,

$$
\left\|g_{\varphi}\right\|=\sup _{\|x\| \leq 1}\left|g_{\varphi}(x)\right|=\sup _{\|x\| \leq 1}\left|g_{\varphi}(x)-g_{\varphi}(0)\right| \leq L(\varphi)=\|\varphi\| .
$$

Theorem 3. For every $x \in c_{0}$,

$$
\|x\|=\sup _{\|\varphi\| \leq 1}\left|g_{\varphi}(x)\right|
$$

Proof. For given $x \in c_{0}$ and $\varepsilon>0$ let $\psi_{\varepsilon} \in c_{0}^{*},\left\|\psi_{\varepsilon}\right\|=1$, be such that $\left|\psi_{\varepsilon}(x)\right|=\|x\|-\varepsilon$. Such a functional $\psi_{\varepsilon}$ exists according to the Hahn-Banach Theorem. Let $\psi_{\varepsilon}\left(e_{n}\right)=b_{n}$ and $\gamma: \mathbb{Z}_{0} \rightarrow \mathbb{N}$ be a map such that $\gamma(k)=j$ if $g_{k}(x)=x_{j}$. Clearly, $\gamma$ is a bijection from $\operatorname{supp}(\widehat{x})$ to $\operatorname{supp}(x)$. Let us define a functional $\varphi_{\varepsilon} \in c_{0}\left(\mathbb{Z}_{0}\right)^{*}$ so that $c_{k}=b_{\gamma(k)}, k \in \mathbb{Z}_{0}$. Then $\left\|\varphi_{\varepsilon}\right\| \leq 1$ and

$$
\left|g_{\varphi_{\varepsilon}}(x)\right|=\left|\varphi_{\varepsilon}(\widehat{x})\right|=\left|\psi_{\varepsilon}(x)\right|=\|x\|-\varepsilon .
$$

Since it is true for every $\varepsilon>0$, the required equality holds.

Note that functionals $g_{\varphi}$, where $\varphi \in c_{0}\left(\mathbb{Z}_{0}\right)^{*}$, does not cover all symmetric Lipschitz functions on $c_{0}$. It is known [19, p. 16] that if $f$ and $h$ are Lipschitz functions on a metric space, then both $\max (f(x), h(x))$ and $\min (f(x), h(x))$ are Lipschitz functions with Lipschitz constants bounded by $\max (L(f), L(h))$.

Example. Let $f(x)=\max \left(g_{1}(x), 2 g_{2}(x)\right)$. Then $f$ can not be represented in the form $g_{\varphi}$. Indeed, if $f=g_{\varphi}$ for some $\varphi \in c_{0}\left(\mathbb{Z}_{0}\right)^{*}$, then, since $f(x)$ depends only on $\widehat{x}_{1}$ and $\widehat{x}_{2}$, it should be of the form $f(x)=c_{1} g_{1}(x)+c_{2} g_{2}(x)$ for some constants $c_{1}, c_{2}$. If $x$ is such that $\widehat{x}_{1}=5, \widehat{x}_{2}=1$, then $f(x)=5$; $y$ is such that $\widehat{y}_{1}=5, \widehat{y}_{2}=2$, then $f(y)=5$; and $z$ is such that $\widehat{z}_{1}=5, \widehat{z}_{2}=3$, then $f(z)=6$. But there are no constants $c_{1}, c_{2}$ which satisfy these conditions. 
Another example of a Lipschitz symmetric function which can not be represented as $g_{\varphi}$ is $x \mapsto\|x\|_{c_{0}}=\max \left(g_{1}(x),-g_{-1}(x)\right)$.

Let us recall that a tropical semiring is the semiring $(\mathbb{R} \cup\{+\infty\}, \oplus, \odot)$, where the operations $\oplus$ and $\odot$ are defined by

$$
a \oplus b=\min (a, b) \text { and } a \odot b=a+b, \quad a, b \in \mathbb{R} \cup\{+\infty\} .
$$

It is known (see, e.g., [15]) that $\mathbb{R} \cup\{+\infty\}$ is actually a semiring, where $\oplus$ plays the role of addition, where $+\infty$ is the zero-element, and $\odot$ plays the role of multiplication. Note that $\max (a, b)=-\min (-a,-b)=a+b-\min (a, b)$. A tropical polynomial of several variables $t_{1}, \ldots, t_{n}$ in $\mathbb{R} \cup\{+\infty\}$ is a function of the form

$$
\begin{aligned}
p\left(t_{1}, \ldots, t_{n}\right) & =a \odot t_{1}^{i_{1}} \ldots t_{n}^{i_{n}} \oplus b \odot t_{1}^{j_{1}} \ldots t_{n}^{j_{n}} \oplus \ldots \\
& =\min \left(a+i_{1} t_{1}+\cdots+i_{n} t_{n}, b+j_{1} t_{1}+\cdots+j_{n} t_{n}, \ldots\right),
\end{aligned}
$$

where the coefficients $a, b, \ldots$ are real numbers and the exponents $i_{1}, j_{1}, \ldots$ are integers. We can see that any tropical polynomial can be represented as minimum of some affine functions. Hence, every tropical polynomial is a Lipschitz function on $\mathbb{R}^{n}$ and a finite composition of tropical polynomials is a Lipschitz function. Note that a composition of tropical polynomials is not a tropical polynomial in the general case. Thus we have the following result.

Proposition 3. Let $g_{\varphi_{1}}, \ldots, g_{\varphi_{n}}$ be Lipschitz functions on $c_{0}$, generated by functionals

$$
\varphi_{1}, \ldots, \varphi_{n} \in c_{0}\left(\mathbb{Z}_{0}\right)^{*}
$$

as in Corollary 2 and $q\left(t_{1}, \ldots, t_{n}\right)$ be a finite composition of tropical polynomials of variables $t_{1}, \ldots, t_{n}$. Then

is a Lipschitz function on $c_{0}$.

$$
Q(x)=q\left(g_{1}(x), \ldots, g_{n}(x)\right), \quad x \in c_{0},
$$

Question. Under which conditions on $c_{0}$ every Lipschitz symmetric function can be approximated by functions of the form (3) uniformly on $c_{0}$ ?

Note that the norm in $c_{0}$ can be written exactly as a composition of tropical polynomials of $g_{1}$ and $g_{-1}$

$$
\begin{aligned}
\|x\|_{c_{0}} & =\max \left(g_{1}(x),-g_{-1}(x)\right)=g_{1}(x)-g_{-1}(x)-\min \left(g_{1}(x), g_{-1}(x)\right) \\
& =g_{1}(x) \odot g_{-1}^{-1}(x) \odot\left(g_{1}(x) \oplus\left(g_{-1}\right)^{-1}\right)^{-1}(x) .
\end{aligned}
$$

Thus, we have represented the Lipschitz symmetric function $x \mapsto\|x\|_{c_{0}}$ in the form (3).

\section{References}

[1] Alencar R., Aron R., Galindo P., Zagorodnyuk A. Algebra of symmetric holomorphic functions on $\ell_{p}$. Bull. Lond. Math. Soc. 2003, 35 (1), 55-64. doi:10.1112/S0024609302001431

[2] Aron R.M., Falcó J., García D., Maestre M. Algebras of symmetric holomorphic functions of several complex variables. Rev. Mat. Complut. 2018, 31 (3), 651-672. doi:10.1007/s13163-018-0261-x

[3] Aron R., Falcó J., Maestre M. Separation theorems for group invariant polynomials. J. Geom. Anal. 2018,28 (1), 393-404. doi:10.1007/s12220-017-9825-0

[4] Aron R., Galíndo P., Pinasco D., Zalduendo I. Group-symmetric holomorphic functions on a Banach space. Bull. Lond. Math. Soc. 2016, 48 (5), 779-796. doi:10.1112/blms/bdw043 
[5] Chernega I., Holubchak O., Novosad Z., Zagorodnyuk A. Continuity and hypercyclicity of composition operators on algebras of symmetric analytic functions on Banach spaces. Eur. J. Math. 2020, 6, 153-163. doi:10.1007/s40879019-00390-z

[6] Chernega I., Galindo P., Zagorodnyuk A. Some algebras of symmetric analytic functions and their spectra. Proc. Edinb. Math. Soc. 2012, 55 (1), 125-142. doi:10.1017/S0013091509001655

[7] Galindo P., Vasylyshyn T., Zagorodnyuk A. Analytic structure on the spectrum of the algebra of symmetric analytic functions on $L_{\infty}$. Rev. R. Acad. Cienc. Exactas Fís. Nat. Ser. A Math. 2020, 114, 56. doi:10.1007/s13398-02000791-w

[8] Gonzaléz M., Gonzalo R., Jaramillo J. Symmetric polynomials on rearrangement invariant function spaces. J. London Math. Soc. 1999, 59 (2), 681-697. doi:10.1112/S0024610799007164

[9] Jawad F., Zagorodnyuk A. Supersymmetric polynomials on the space of absolutely convergent series. Symmetry 2019, 11 (9), 1111. doi:10.3390/sym11091111

[10] Lindestrauss J., Tzafriri L. Classical Banach Spaces I: Sequence Spaces. In: Ergebnisse der mathematik und ihrer Grenzgebiete, 92. Springer-Verlag, Berlin, Heidelberg, New York, 1977.

[11] Macdonald I.G. Symmetric Functions and Orthogonal Polynomials. In: University Lecture Series, 12. AMS, Providence, R.I., 1998.

[12] Martsinkiv M., Zagorodnyuk A. Approximations of symmetric functions on Banach spaces with symmetric bases. Symmetry 2021, 13 (12), 2318. https: / / doi.org/10.3390/sym13122318

[13] Mujica J. Complex Analysis in Banach Spaces. Elsevier, Amsterdam, 1986.

[14] Nemirovskii A.S., Semenov S.M. On polynomial approximation of functions on Hilbert space. Math. USSR Sb. 1973, 21 (2), 255-277. (translation of Math. Sb. 1973, 92(134) (2(10)), 257-281. (in Russian))

[15] Speyer D.E., Sturmfels B. Tropical mathematics. Math. Mag. 2009, 82 (3), 163-173. doi:10.1080/0025570X.2009.11953615

[16] Vasylyshyn T. Symmetric functions on spaces $\ell_{p}\left(\mathbb{R}^{n}\right)$ and $\ell_{p}\left(\mathbb{C}^{n}\right)$. Carpathian Math. Publ. 2020, 12 (1), 5-16. doi:10.15330/cmp.12.1.5-16

[17] Vasylyshyn T.V., Zagorodnyuk A.V. Symmetric polynomials on the Cartesian power of the real Banach space $L_{\infty}[0,1]$. Mat. Stud. 2020, 53 (2), 192-205. doi:10.30970/ms.53.2.192-205

[18] Vasylyshyn T., Zagorodnyuk A. Continuous symmetric 3-homogeneous polynomials on spaces of Lebesgue measurable essentially bounded functions. Methods Funct. Anal. Topology 2018, 24 (4), 381-398.

[19] Weaver N. Lipschitz Algebras. World Scientific, New Jersey, 1999.

Received 02.02.2021

Revised 03.11.2021

Марцінків М.В., Василишин С.І., Василишин Т.В., Загороднюк А.В. Аіпиицеві симетричні фрункиї̈ на банахових просторах з симетричним базисом // Карпатські матем. публ. - 2021. - Т.13, №3. - C. 727-733.

Аосліджено ліпшицеві симетричні функції на банаховому просторі $X$ з симетричним базисом. Розглянуто степеневі симетричні поліноми на $\ell_{1}$ i показано, що вони є ліпшицевими у необмеженій області, яка складається з векторів $x \in \ell_{1}$ координати яких $\left|x_{n}\right| \leq 1$. Використовуючи функції max та min і тропічні поліноми від кількох змінних, побудовано широкий клас ліпшицевих симетричних функцій на банаховому просторі $c_{0}$, який можна описати як напівкільце композицій тропічних поліномів над простором $c_{0}$.

Ключові слова і фрази: Аіпшицева симетрична аналітична функція на банаховому просторі, симетричний базис, тропічний поліном. 